\title{
SMART PHONE BASED ENERGY MANAGEMENT SYSTEM USING RASPBERRY PI3
}

\author{
Dr. Dhiraj Sunehra \\ Department of Electronics \& Communication Engineering \\ Faculty of Engineering \& Technology \\ Jawaharlal Nehru Technological University Hyderabad, Hyderabad, India
}

\begin{abstract}
In many places, energy wastage arises when a person leaves the home or office without turning off the home appliances such as lights and fans. Due to the negligence of the person, lot of energy is wasted unused and results in higher amount of electricity bill. Nowadays, almost every household member has a smart phone because of their affordable prices. In this paper, an energy management system is developed using Smart phone's Bluetooth technology. The proposed system is built on Raspberry Pi3 processor and uses the Bluetooth communication in the user's phone to determine whether the user is at home or not. It is not necessary for the user's phone to be in discoverable mode for its functionality, it should be just turned on. When the user is out of homeloffice, the Bluetooth device in the phone gets unpaired from the energy management system's Bluetooth device. This in turn turns off the unused devices such as lights and fans to save energy. Multiple Bluetooth devices can also be used in case of multiple users. The user can control the appliances manually through switches when at home.
\end{abstract}

Key words: Raspberry Pi3, Energy Management, Smart phone, Bluetooth technology

Cite this Article: Dr. Dhiraj Sunehra, Smart Phone Based Energy Management System Using Raspberry Pi3, International Journal of Advanced Research in Engineering and Technology, 10(2), 2019, pp 463-471.

http://iaeme.com/Home/issue/IJARET?Volume=10\&Issue $=2$

\section{INTRODUCTION}

Electric power is a basic necessity in every home, office or industry. With the growth of technology, the need for electricity has increased. Electricity is necessary to power on our computers, lights, fans, air conditioners, washing machines, water heaters, street lights and many more. We have numerous applications, but we produce limited electric power. Since the generation of greater electric power to meet the demand is always challenging, an easier solution would be to manage the available electric power efficiently. The energy management deals with the conservation of resources and our concern is the electric power. Energy management also has an added advantage of reduced resource cost due to increase in efficient usage and decrease in wastage. 
As the technology advances, more and more devices are brought home by a person, which requires power to operate. In the recent years, several systems have been designed for efficient energy management. But these systems are expensive and are not easy to install. Because of this, people do not opt for such systems for their homes. To overcome such problems, a simple and cost-effective system is implemented that can be easily upgraded. This design uses the Raspberry Pi processor along with a Bluetooth dongle. The credit card sized computer offers low cost and compact size, whereas the Bluetooth offers very low power consumption [1].

\section{HARDWARE DESCRIPTION}

\subsection{Block Diagram}

Figure 1 shows the block diagram of smart phone-based energy management system. It consists mainly three blocks. Firstly, the Raspberry Pi3 board acts as the heart of the system. It is programmed using Python language. When the program executes, the Raspberry Pi decides when and what device to turn on. The Raspberry Pi is connected to the Bluetooth module, power supply and the relay board. Secondly, the USB Bluetooth dongle is connected to the Raspberry Pi using the female ports available on the Raspberry Pi. The USB Bluetooth dongle should be Bluetooth 2.0 or greater. Thirdly, the relay board is necessary to control the electrical appliances. The relay board provides magnetic isolation between the Raspberry Pi and the mains supply. The mains power supply can be $110-240 \mathrm{~V}$.

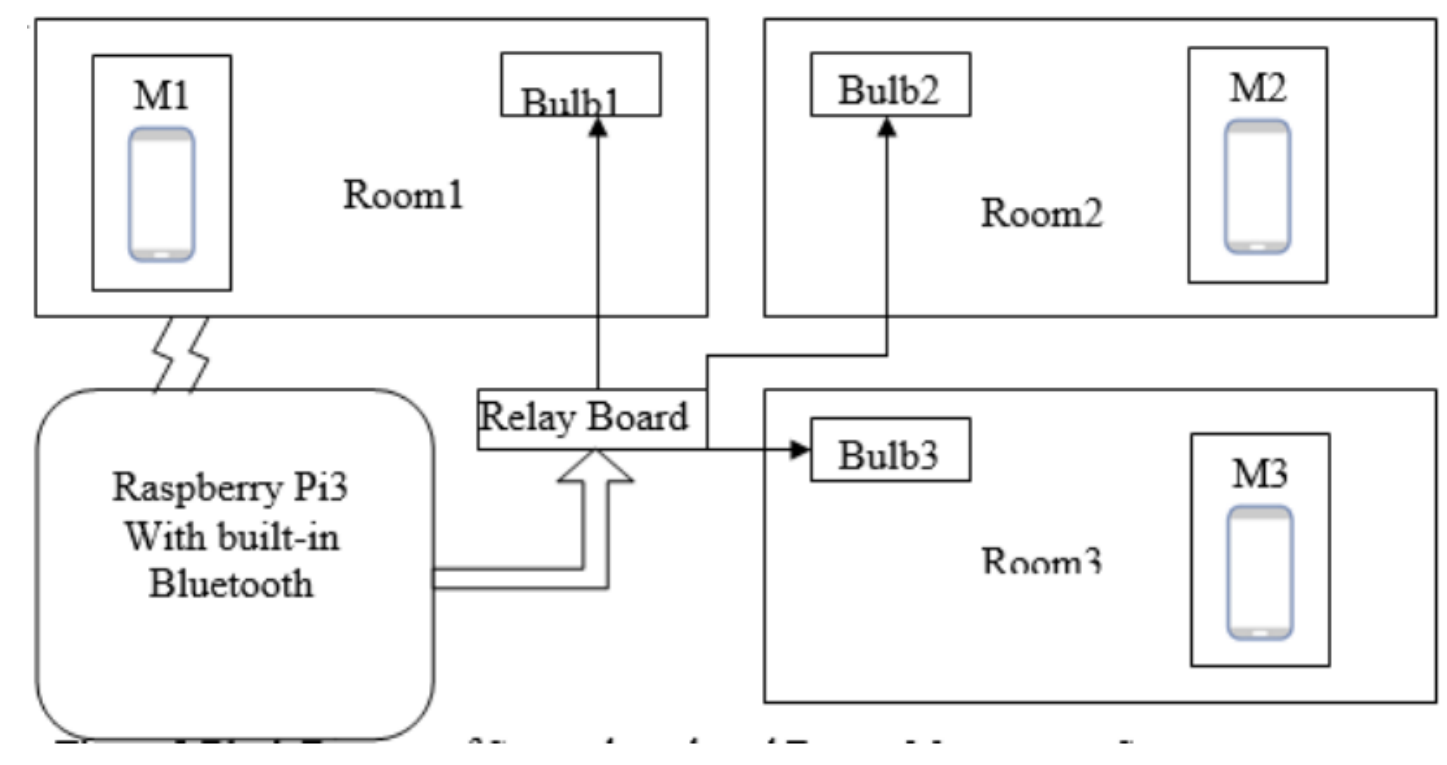

Figure 1 Block Diagram of Smart phone-based Energy Management System

\subsection{Raspberry Pi3 Model B Board}

Raspberry $\mathrm{Pi}$ is a minicomputer, which is a credit card sized board. It has a Broadcom BCM2837 system on a chip (SoC), 1.2GHz processor, Video Core IV GPU and 1GB RAM. It has 40 GPIO pins, one 10/100M Ethernet port, 4 USB ports, HDMI 1.4 composite video port and a four-pole stereo audio connector (Figure 2) [2]. There is no inbuilt storage in Raspberry Pi. The operating system for the Raspberry Pi is loaded into the SD card whose storage can range from $8 \mathrm{~GB}$ to $64 \mathrm{~GB}$. The Microchip LAN9512 LAN controller chip controls the Ethernet port. Camera Serial Interface (CSI) connector is used to connect digital camera to the Raspberry Pi. HDMI port is used to connect a monitor or display to Raspberry Pi so that we can view the working environment. The Raspberry Pi3 board has 40 pins out of which 26 are GPIO pins and remaining are Vcc and Ground. This pins act as the medium of interface between Pi and external 
devices. There are two I2C pins for serial communication. There is an inbuilt Wi-Fi module to connect to internet along with Ethernet port.

Raspberry Pi requires a $+5 \mathrm{~V}$ DC power supply adapter that supply atleast $700 \mathrm{~mA}$ to power on.

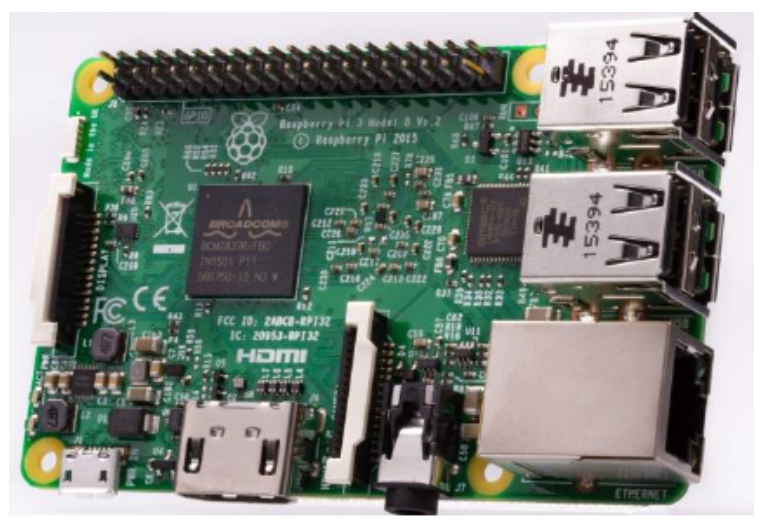

Figure 2 Raspberry Pi3 Model B Board

\subsection{Relay}

A relay is usually an electromechanical device that is actuated by an electrical current. The current flowing in one circuit causes the opening or closing of another circuit. Relays are like remote-control switches and are used in many applications because of their relative simplicity, long life, and proven high reliability. Relays are used in a wide variety of applications throughout industry, such as in telephone exchanges, digital computers and automation systems. Highly sophisticated relays are utilized to protect electric power systems against trouble and power blackouts as well as to regulate and control the generation and distribution of power.

\section{SOFTWARE TOOLS}

Python is a widely used general-purpose, high-level programming language. Its design philosophy emphasizes code readability, and its syntax allows programmers to express concepts in fewer lines of code than would be possible in languages such as $\mathrm{C}++$ or Java [3]. The language provides constructs intended to enable clear programs, including object-oriented, imperative and functional programming or procedural styles. It features a dynamic type system and automatic memory management and has a large and comprehensive standard library.

PuTTY is a free and open emulator, serial console and network file transfer application. It supports several network protocols, including SCP, SSH, Telnet, rlogin, and raw socket connection [4]. It can also connect to a serial port. The name "PuTTY" has no definitive meaning. PuTTY was originally written for Microsoft Windows but it has been ported to various other operating systems. Official ports are available for some Unix-like platforms, with work-in-progress ports to Classic Mac OS and Mac OSX, and unofficial ports have been contributed to platforms such as Symbian, Windows Mobile and Windows Phone. PuTTY was written and is maintained primarily by Simon Tatham and is currently beta software. ExtraPuTTY is a software tool used to access Raspberry Pi.

\section{SCHEMATIC DIAGRAM}

Figure 3 shows the schematic diagram of the energy management system. Three bulbs are connected to three relay boards. All relay boards are given with power supply in series. Relay 1 is connected to the GPIO3. Relay 2 is connected to the GPIO14. Relay 3 is connected to the 
GPIO15. Raspberry Pi board is given with DC power supply. Remaining pins of all the relays are grounded.

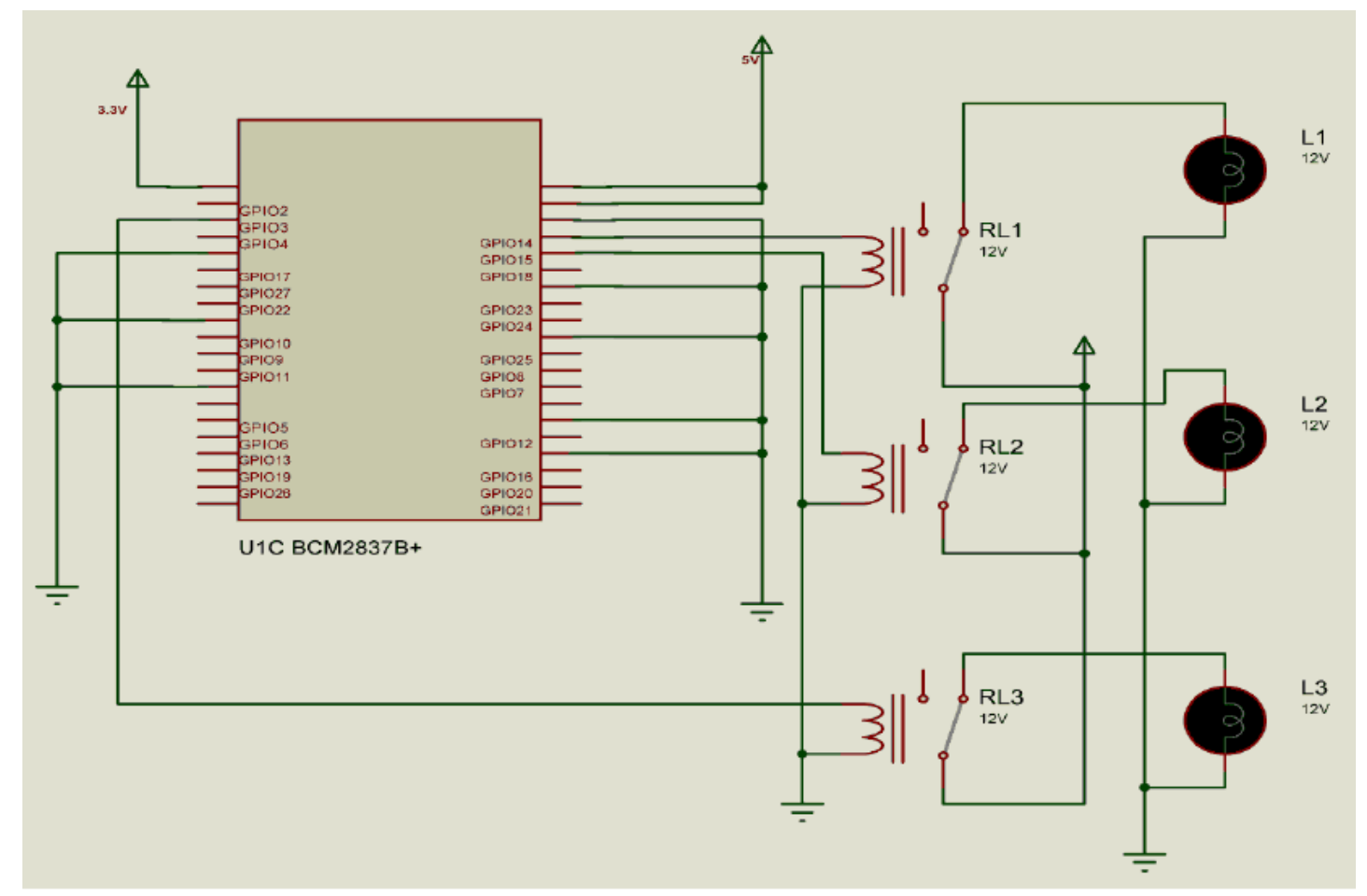

Figure 3 Schematic Diagram of Energy Management System

\section{FLOWCHART}

Figure 4 shows the flowchart of the sequence of events that occur in the energy management system. 


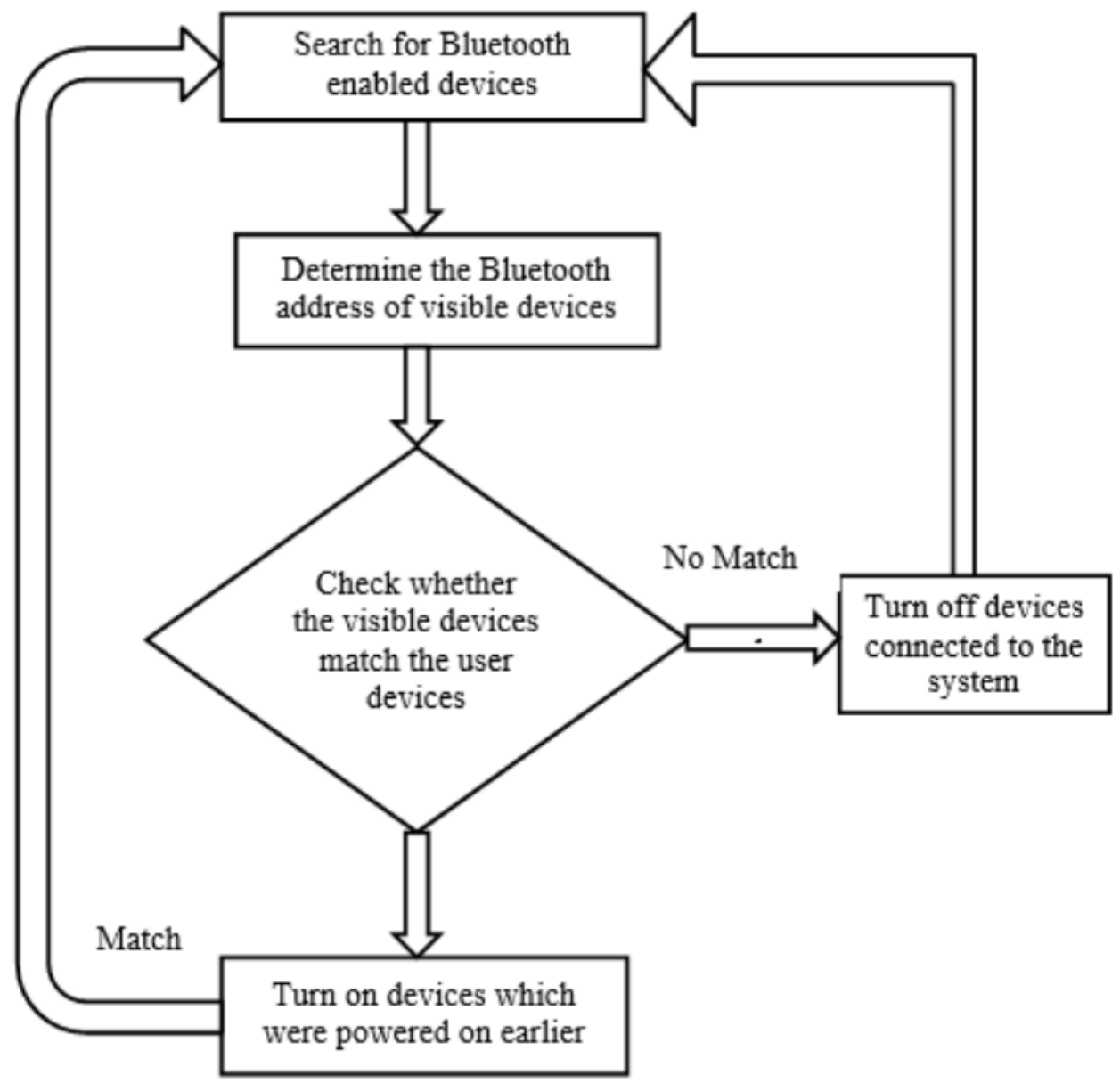

Figure 4 Flowchart of Smart phone-based Energy Management System

\section{EXPERIMENTAL RESULTS}

Figure 5-8 shows the experimental results obtained when various smart phones are within the pairing range. The setup of smart phone-based energy management system consists of Raspberry Pi with Bluetooth dongle, three relays with bulbs. Three smart phones of Moto make, Redmi make and Vivo make are chosen in the experiment. Figure 5 shows the experimental setup with Bulb $1 \mathrm{ON}$ which is paired with Moto make smart phone. The Bulb 2 and Bulb 3 are OFF as they are not paired with any phones. It means the users of Redmi phone and Vivo phone is not in the pairing range of the Bluetooth module of Raspberry Pi (User not at home / office). Figure shows the setup with Bulb $2 \mathrm{ON}$ which is paired with Redmi make smart phone. The Bulb 1 and Bulb 3 are OFF as they are not paired with any phones. Figure 7 shows the setup with Bulb $3 \mathrm{ON}$ which is paired with Vivo make smart phone. The Bulb 1 and Bulb 2 are OFF as they are not paired with any phones. Figure 8 shows the setup with Bulb 1 and Bulb 2 ON which are paired with Moto and Redmi make smart phones. Bulb 3 is OFF as the user of Vivo make is not in the Bluetooth pairing range. 


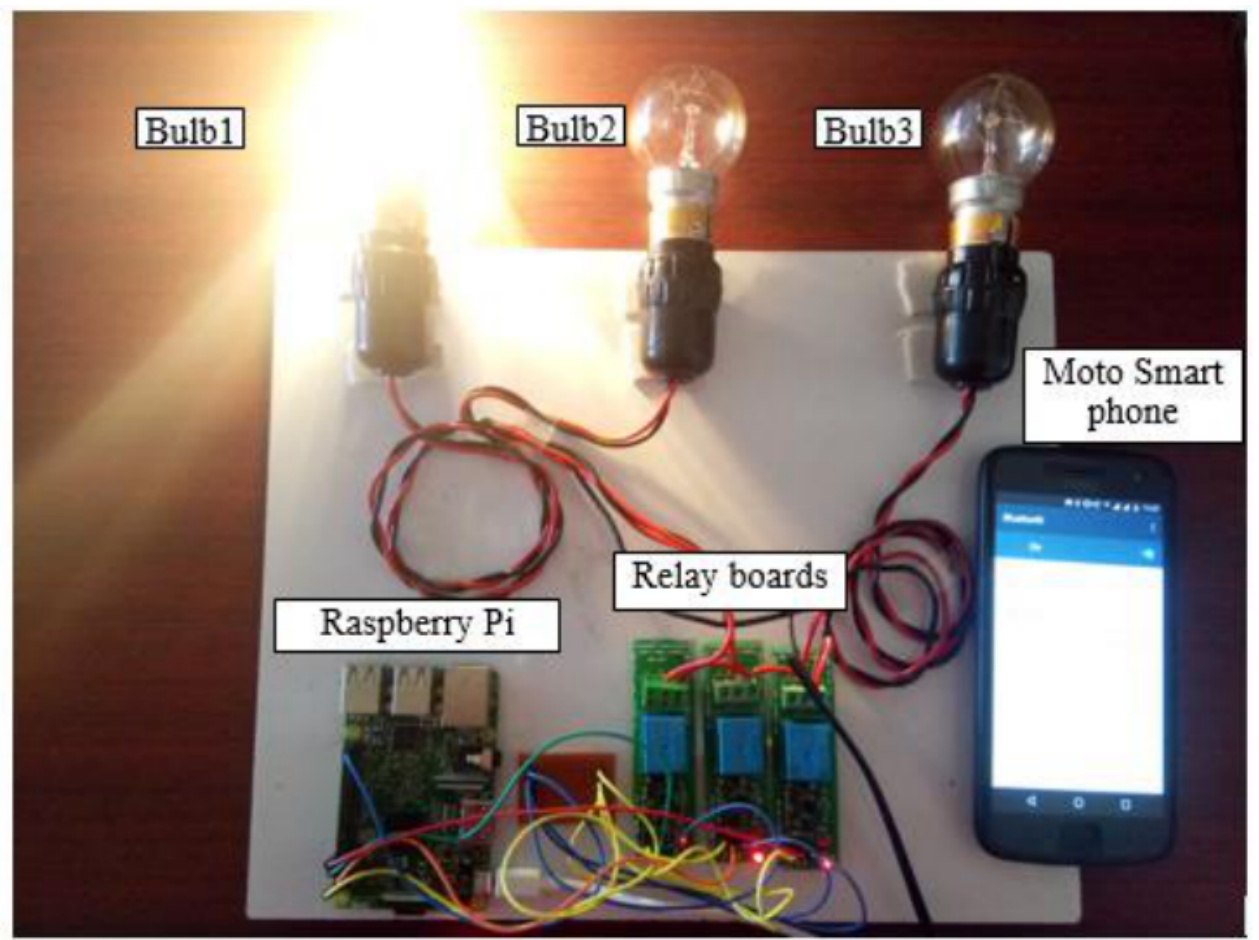

Figure 5 Experimental Setup showing Bulb 1 ON which is paired with Moto make Smart phone (Bulb 2 and 3 are OFF)

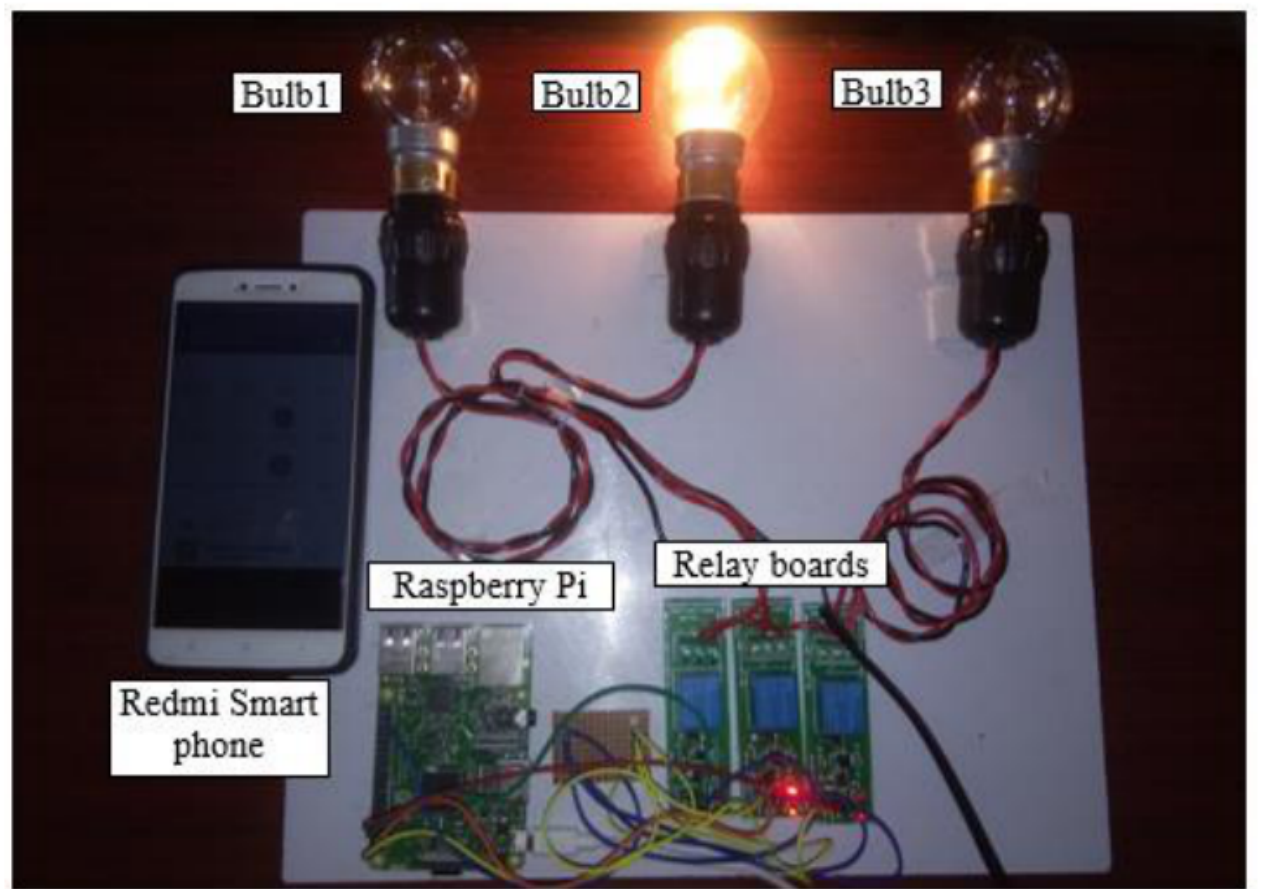

Figure 6 Experimental Setup showing Bulb 2 ON which is paired with Redmi make Smart phone (Bulb 1 and 3 are OFF) 


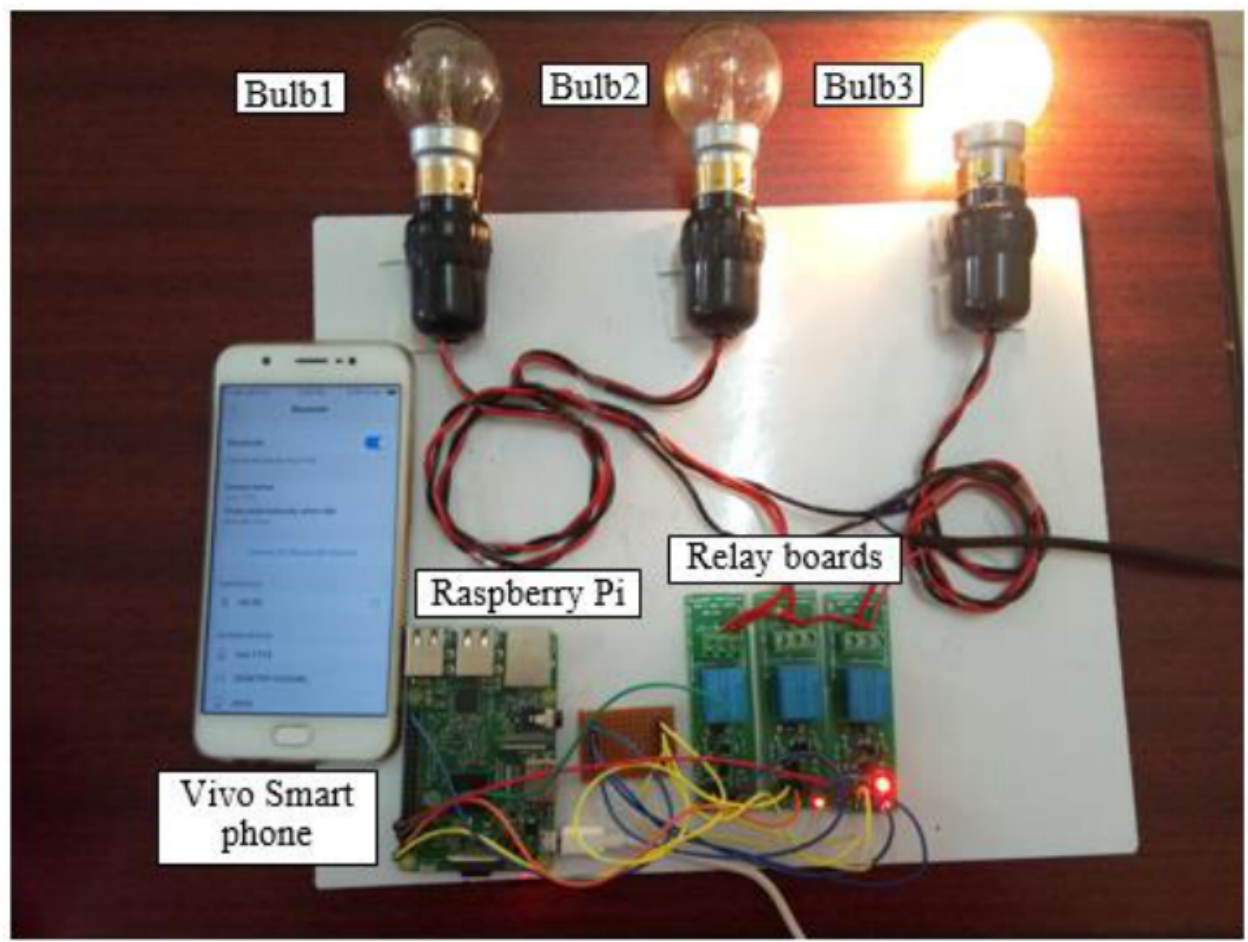

Figure 7 Experimental Setup showing Bulb 3 ON which is paired with Vivo make Smart phone (Bulb 1 and 2 are $\mathrm{OFF}$ )

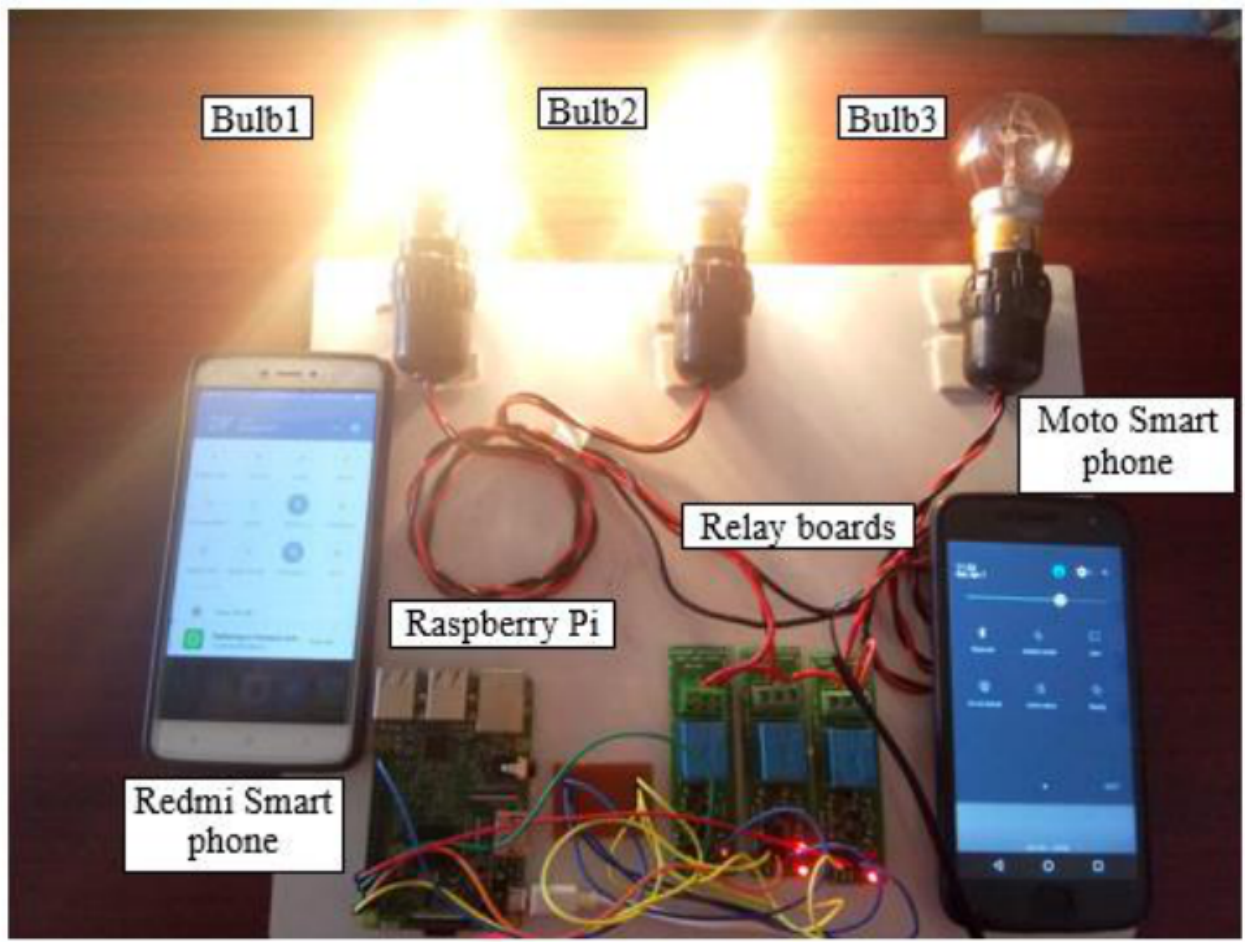

Figure 8 Experimental Setup showing Bulb 1 and 2 ON which are paired with Moto and Redmi make Smart phone (Bulb 3 is OFF)

Figure 9-12 shows the corresponding outputs displayed in PuTTY window. Observe that the value returned is ' 0 ' when a smart phone is paired or detected. Value 256 is returned when a smart phone is not detected. This in turn activates the relay to switch the corresponding bulbs $\mathrm{ON}$. 


\section{Dr. Dhiraj Sunehra}

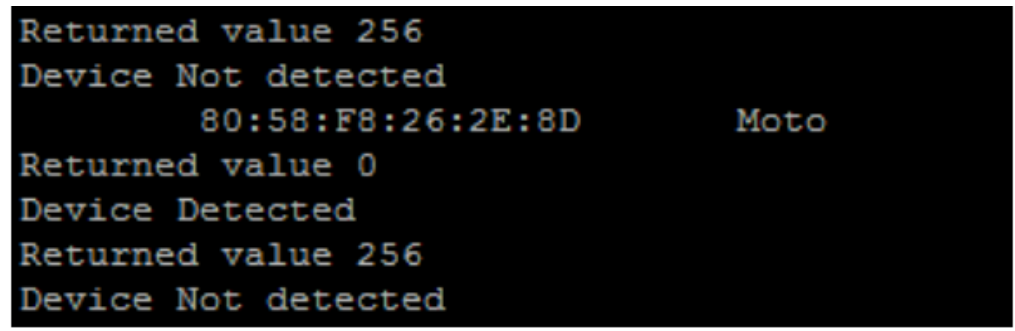

Figure 9 PuTTY window output when Moto make Smart phone is detected

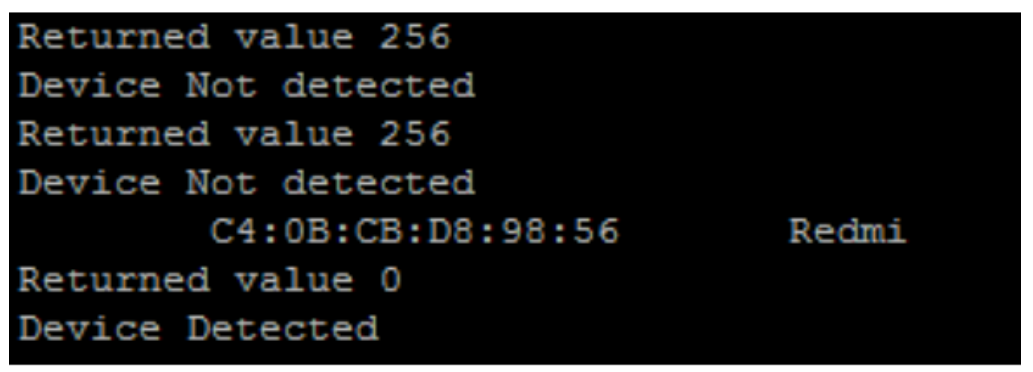

Figure 10 PuTTY window output when Redmi make Smart phone is detected

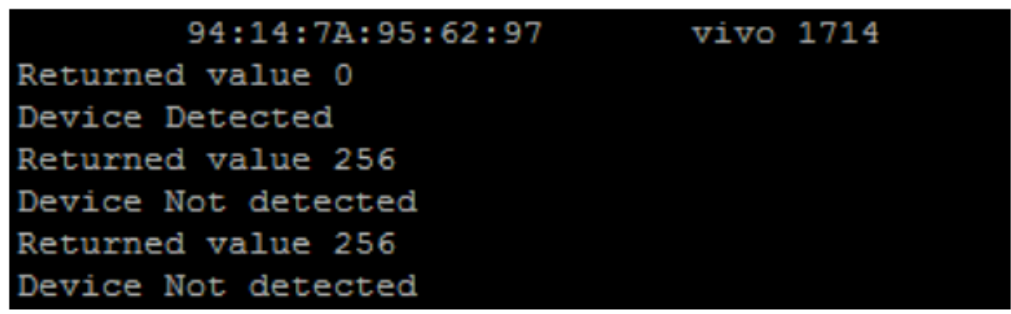

Figure 11 PuTTY window output when Vivo make Smart phone is detected

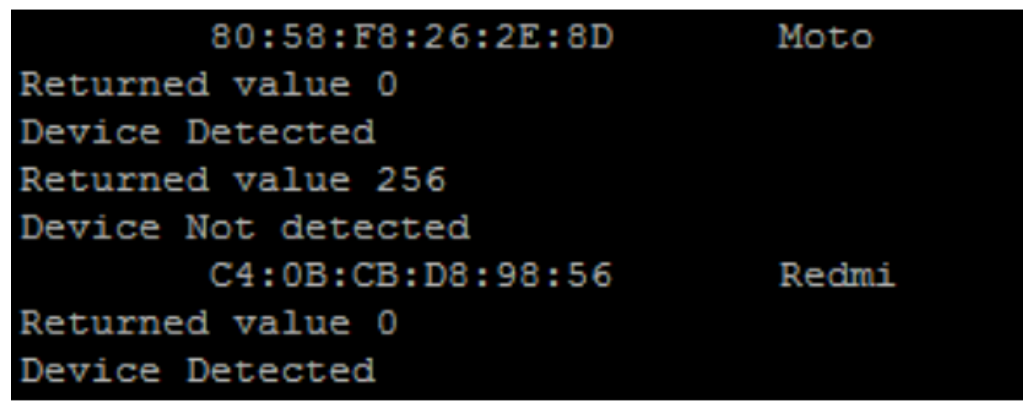

Figure 12 PuTTY window output when Moto and Redmi make Smart phones are detected

\section{CONCLUSIONS}

In this paper, a smart phone-based energy management system is implemented using Bluetooth technology. This system helps in reducing the unnecessary power consumption across the globe. With the development of technology, people carry multiple Bluetooth enabled devices such as the mobile phone and fitness band. This system is not restricted to only mobile phones. People who wear fitness bands to track their daily activity can use the Bluetooth address of the device to control the system. The user can turn off their Bluetooth device or devices when they are about to sleep to turn off any devices which was left turned on. Since most of the electrical appliances are turned off in the absence of the user, the accidents due to electrical fires are minimized. This system not only helps to save energy but also improves safety. The use of Raspberry Pi helps in keeping the system cost low, yet allowing it to be upgraded. 


\section{REFERENCES}

[1] J.C. Haartsen and S. Mattisson, "Bluetooth - a new low-power radio interface providing shortrange connectivity", Proc. of the IEEE, Vol. 88, Issue 10, Oct. 2000, pp. 1651 - 1661, IEEE.

[2] https://www.raspberrypi.org/

[3] Richard L. Halterman, "Learning to Program with Python”, 2011 (www.cs.uky.edu/).

[4] https://en.wikipedia.org/wiki/PuTTY.

[5] Kapil K Shukla, Kaushik I Manavadariya and Deven J Patel, Comparative Study of Bluetooth, 802.11 and Hiperlan, International Journal of Computer Engineering and Technology (IJCET), Volume 4, Issue 3, May-June (2013), pp. 455-463

[6] Nilima Bargal and Pratima Bhalerao, Effortless Sharing of Books on E-Library Through Bluetooth, International Journal of Electronics and Communication Engineering \& Technology (IJECET), Volume 5, Issue 6, June (2014), pp. 61-66

[7] D. Sobya, S. Nallusamy and Partha Sarathi Chakraborty, Establishment of Smart Meter Reading Scheme for Monitoring the Power In Residence Using Bluetooth. International Journal of Electrical Engineering \& Technology, 9(1), 2018, pp. 67-75.

[8] Gemechu Wako Samu and Prachi Kadam, Survey on Indoor Localization: Evaluation Performance of Bluetooth Low Energy and Fingerprinting Based Indoor Localization System. International Journal of Computer Engineering \& Technology, 8(6), 2017, pp. 23-35. 